\title{
Kernos
}

Revue internationale et pluridisciplinaire de religion grecque antique

$26 \mid 2013$

Varia

\section{J. Rasmus Brandt, Jon W. Iddeng (éd.), Greek and Roman Festivals. Content, Meaning, and Practice}

Vinciane Pirenne-Delforge

\section{(2) OpenEdition \\ Journals}

Édition électronique

URL : http://journals.openedition.org/kernos/2152

DOI : 10.4000/kernos.2152

ISSN : 2034-7871

Éditeur

Centre international d'étude de la religion grecque antique

Édition imprimée

Date de publication : 10 octobre 2013

Pagination : 401-402

ISSN : 0776-3824

Référence électronique

Vinciane Pirenne-Delforge, « J. Rasmus Brandt, Jon W. Iddeng (éd.), Greek and Roman Festivals.

Content, Meaning, and Practice », Kernos [En ligne], 26 | 2013, mis en ligne le 10 octobre 2013,

consulté le 02 mars 2021. URL : http://journals.openedition.org/kernos/2152 ; DOI : https://doi.org/

$10.4000 /$ kernos. 2152

Ce document a été généré automatiquement le 2 mars 2021.

Kernos 


\title{
J. Rasmus Brandt, Jon W. Iddeng (éd.), Greek and Roman Festivals. Content, Meaning, and Practice
}

\author{
Vinciane Pirenne-Delforge
}

\section{RÉFÉRENCE}

J. Rasmus Brandt, Jon W. Iddeng (éd.), Greek and Roman Festivals. Content, Meaning, and Practice, Oxford, Oxford University Press, 2012.1 vol. $14,5 \times 22 \mathrm{~cm}$, xvii +405 p. ISBN : 978-0-19-969609-3.

1 Il s'agit d'un très bel ensemble de contributions issu d'un colloque tenu en 2006 près de Bergen, en Norvège. Le temps mis à paraître est sans doute un des rares défauts de ce bouquet d'études qui abordent sous divers angles la question des "fêtes" dans l'antiquité gréco-romaine. Outre la production d'un index remarquablement détaillé, les deux éditeurs ont fait le nécessaire pour donner une cohérence au propos général du volume : le premier livre une intéressante introduction qui tire les lignes de force de l'ensemble et le second pose la question de savoir « What is a Graeco-Roman Festival? ». La réponse s'amorce grâce à ce que J.W. Iddeng appelle «a polythetic approach » : il met en place une série de critères dont tous ne doivent pas forcément se retrouver en même temps dans un contexte donné, mais dont la convergence d'un certain nombre permet de parler de «fête ». On trouve, parmi ces critères, le caractère cyclique de la célébration, un lieu donné, le caractère public de l'événement (même si une partie reste secrète), la mise en place d'actes de culte (mais pourquoi parler de "pagan worship » ?) qui articulent à la fois la relation au monde des dieux et construit la communauté des célébrants. - W. Burkert adopte lui aussi un point de vue global dans un bel article embrassant d'un même regard, tout en nuances, une koinè de la Méditerranée orientale pour parler de célébrations festives que les anciens ont déjà partiellement théorisées (e.g. Platon et Strabon). - S. des Bouvrie, quant à elle, s'attaque à l'arrière-plan conceptuel de l'analyse des fêtes où elle convoque les théories anthropologiques de la 
culture et des développements qu'elle a déjà réservés antérieurement à la question du "symbole» et des démarches "non rationnelles» (mais pas "irrationnelles») qu'il implique. Elle tente ensuite d'appliquer les outils collectés aux fêtes d'Olympie (pour Zeus et pour Héra, selon Pausanias) et aux Grandes Dionysies d'Athènes. - G. Ekroth s'attaque elle aussi à des célébrations tenues à Olympie, mais en l'honneur de Pélops. L'ambition de cette étude dépasse largement celle de l'étude des Olympia et des Heraia de l'article précédent puisqu'il s'agit de reconstituer, sur la base d'un dossier archéologique minutieusement analysé et de témoignages littéraires, l'histoire du culte de Pélops sur le site. Le spectre chronologique va de l'introduction du héros en ce lieu, probablement au $\mathrm{VI}^{\mathrm{e}} \mathrm{s}$. (l'hypothèse d'un culte antérieur est définitivement enterrée) jusqu'à la période romaine, dont date le témoignage de Pausanias, essentiel dans ce dossier. Ce travail minutieux deviendra assurément un passage obligé pour tous ceux qu'intéressent le héros et le site d'Olympie. On soulignera, notamment, l'hypothèse faite par l'A. du statut de "national hero » des Éléens (p. 112) que Pélops aurait acquis $\mathrm{au} \mathrm{V} \mathrm{V}^{\mathrm{e}} \mathrm{s}$. (on résistera toutefois à la notion de "poliad cult», p. 118). La construction d'un mur de protection du péribole peut constituer un indice d'une célébration réservée aux Éléens, au cœur du sanctuaire panhellénique, à l'instar du culte rendu par les Déliens au héros Anios dans leur île ouverte sur le monde égéen. - J. Rasmus Brandt, un des éditeurs du volume, aborde quant à lui un problème complexe, à savoir la possibilité de reconstituer la forme et le contenu de fêtes quand on ne dispose que d'un matériel strictement archéologique. Il s'agit de déterminer une série de critères permettant de traiter avec le plus de rigueur possible les données des fouilles, notamment quand la publication en est ancienne et ne répond pas forcément aux standards de rigueur actuels. Cet article exploratoire rassemble les données chiffrées des trouvailles de 10 sites pour déterminer les données de la culture matérielle qui permettent de distinguer le contenu d'une fête (sa fonction sociale et les représentations à l'œuvre, notamment) et sa forme (la manière dont cette fonction est exprimée au cours de la célébration), tout en tenant suffisamment compte de la diachronie et d'éventuelles évolutions. Au final, le lecteur reste un peu sur sa faim (" ancient festivals appear to be a public concern through which a constant dialogue with the deities was maintained to secure - in a broad sense - the protection, preservation, and growth of the society ", p. 178). Mais il s'agit d'un appel très lucide à améliorer toujours davantage la qualité de précision des rapports de fouille. - J. Neils ouvre son article sur les Panathénées par une très jolie comparaison avec une fête florentine en 1440 pour se concentrer ensuite sur l'ancrage civique perceptible dans la frise du Parthénon et les évolutions de la fête, sur ce plan, au cours $d u V^{e} s$. S. Scullion, à grand renfort de citations d'orateurs athéniens, interroge le statut du chœur tragique et de ce qui devient, de manière générique, des kuklikoi choroi, pour conclure sur le constat que tous les éléments d'une fête n'étaient pas « rituels » au sens strict et non forcément ressentis comme tels. Pour l'A., la dichotomie "sacré / profane " peut être une manière de rendre compte du fait que la tragédie, au IV ${ }^{\mathrm{e}} \mathrm{s}$., n'était plus nécessairement considérée comme une forme de rituel religieux. $K$. Buraselis intègre la figure des souverains hellénistiques et des empereurs romains à cette réflexion sur les fêtes. Il s'agit d'une réflexion importante, venant nourrir un débat très actuel, sur le statut des honneurs rendus à ces humains mortels dans le cadre d'« appendices » ajoutés aux fêtes traditionnelles. - Ces dossiers grecs sont suivis de quatre articles sur des fêtes romaines: Chr.J. Smith tente de débrouiller l'écheveau complexe des attestations sur les Féries latines; J. Scheid analyse les fêtes célébrées 
dans l'espace du Forum Boarium et déploie les significations implicites qui forment le discours sous-jacent à ces rituels; J. Rüpke interroge les changements qui ont dû advenir dans la célébration des fêtes républicaines, tout en soulignant avec vigueur que les dieux n'étaient pas des éléments accessoires de ce type de rituel («Religious occasions meant actions involving the gods ", p. 315); M. Beard décortique les lectures possibles du culte de la Mater Magna introduite à Rome au terme de la "guerre d'Hannibal », et montre notamment combien le témoignage du chrétien Prudence sur le prétendu Taurobolium doit être replacé dans son contexte narratif.

\section{AUTEURS}

VINCIANE PIRENNE-DELFORGE

F.R.S.-FNRS - Université de Liège 